LOVERA, L. H.; LIMA, E. S.; MONTANARI, R.; TOMAZ, P. K.; ROSA, P. A. L. Atributos químicos de um cambissolo por meio de análise multivariada na cultura da pupunha. Revista de Agricultura Neotropical, Cassilândia-MS, v. 1, n. 2, p. 8-16, out./dez. 2014.

\title{
ATRIBUTOS QUÍMICOS DE UM CAMBISSOLO POR MEIO DE ANÁLISE MULTIVARIADA NA CULTURA DA PUPUNHA
}

\author{
LENON HENRIQUE LOVERA ${ }^{1}$, ELIZEU DE SOUZA LIMA ${ }^{1}$, RAFAEL \\ MONTANARI ${ }^{1}$, PAMELA KERLYANE TOMAZ ${ }^{1}$, POLIANA APARECIDA LEONEL \\ ROSA $^{1}$ \\ ${ }^{1}$ UNESP/Ilha Solteira, SP, Brasil, lenon_lovera@hotmail.com, elizeu.florestal@gmail.com, montanari@agr.feis.unesp.br, \\ pamelazootecnia@hotmail.com, polirosa1@ hotmail.com
}

RESUMO: Por se tratar de um cultivo perene, a pupunheira se torna bastante exigente quanto às propriedades físicas e químicas do solo, sendo que os atributos químicos do solo refletem de forma mais imediata ao efeito causado pelo manejo que é adotado. O objetivo do presente trabalho foi identificar por meio de análises multivariadas os atributos químicos do solo que melhor explicam a variabilidade da cultura da pupunha em Registro, SP. Em 2014, no município de Registro (SP), foi analisado os componentes produtivos da cultura da pupunha em função de alguns atributos químicos de um Cambissolo eutrófico. Foi demarcada a malha geoestatística, com 54 pontos amostrais, numa área de 1 ha. A classificação em grupos foi feita por três métodos: método de agrupamentos hierárquico, método não-hierárquico k-means e análise de componentes principais. Pode se concluir que a análise multivariada por meio da análise de componentes principais permitiu identificar dois grupos que explicam 56,62\% da variabilidade total dos dados, onde estes grupos são constituídos pelos atributos químicos matéria orgânica, potássio, magnésio, alumínio, capacidade de troca catiônica e saturação por alumínio se destacassem como componentes principais que se mostraram com maior poder de explicação da variação do diâmetro do caule e o número de perfilhos da pupunha.

PALAVRAS-CHAVE: Fertilidade do solo, multivariada, componentes principais

\section{CHEMICAL PROPERTIES ON AN EUTROCHREPT BY MULTIVARIATE ANALYSIS IN THE CULTURE OF PEACH PALM}

\begin{abstract}
Because it is a perennial crop, the peach palm becomes quite demanding on the soil physical and chemical properties and the soil chemical properties reflect more immediately the effect caused by the adopted management. The objective of this study was to identify by multivariate chemical analyzes of soil attributes that best explain the peach palm crop variability. In 2014, in the municipality of Registro (Sao Paulo State), was analyzed the productive components of peach palm crop due to some chemical attributes on an eutrophic Eutrochrept (Cambisol). The geostatistical grid was marked, with 54 sampling points, in an area of 1 ha. The classification into groups was made by three methods: hierarchical groupings method, non-hierarchical k-means method and principal component analysis. So it can be concluded that the multivariate analysis using principal component analysis allowed us to identify two groups that explain $56.62 \%$ of the total variability of the data, where these groups are constituted by the chemical attributes: organic matter, potassium, magnesium, aluminum, capacity cation exchange and aluminum saturation stood out as the major components that demonstrated greater explanatory power of the stem diameter variation and the number of tillers from the peach palm.
\end{abstract}

KEY WORDS: Soil fertility, multivariate, main, components. 


\section{INTRODUÇÃO}

O cultivo da pupunha (Bactris gasipaes Kunth) para a produção de palmito vem despertando interesse desde a década de 70 , sendo uma nova opção em substituição aos tradicionais Euterpe spp, tendo em vista as qualidades agronômicas, ecológicas, industriais e comerciais. A região Sudeste concentra a maior área cultivada e colhida de palmito (em torno de $40 \%$ do total) e o Estado de São Paulo destaca-se como um dos maiores produtores brasileiros de pupunha, com área plantada de 8.580 ha, área colhida de 3.120 ha e produção de 9.220 ton, principalmente na região do vale do Ribeira, que compreende os munícipios de Registro, Iguape, Sete Barras, Jacupiranga, Cajati e Juquiá (IBGE, 2009).

Por se tratar de um cultivo perene, a pupunheira se torna bastante exigente quanto às propriedades físicas e químicas do solo (BOVI, 2000). Os atributos químicos do solo refletem de forma mais imediata ao efeito causado pelo manejo que é adotado. Além disso, permitem uma melhor compreensão da dinâmica dos elementos considerados nutrientes para as plantas e organismos do solo. Lourente et al. (2011) verificaram que a substituição da vegetação nativa por sistemas de cultivo pode causar importantes alterações nos atributos químicos do solo, já no primeiro ano de implantação. A adoção de práticas conservacionistas, também tem efeito positivo sobre o teor e a qualidade da matéria orgânica do solo, refletindo direta ou indiretamente sobre as características químicas do solo (FRAZÃO et al., 2008).

Com o emprego das técnicas multivariadas é possível descrever a similaridade (análise de agrupamentos) em sistema de cultivo, como também descrever as variáveis da química do solo que explicam a maior variabilidade possível em um conjunto de dados (componentes principais) (CORTEZ et al., 2011).

Uma das técnicas multivariadas mais utilizadas é a análise de agrupamento, que pode ser complementada pela análise de componentes principais (CP1 e CP2), cuja finalidade é explicar a estrutura dos dados, mediante processo matemático que preserva a maior parte das informações fornecida pelas variáveis originais (VALENTIM, 2000).

O objetivo da análise de agrupamento é dividir um conjunto original de dados em vários grupos, segundo critérios de similaridade ou dissimilaridade, sendo que $\mathrm{o}$ dendrograma, componente da análise de agrupamento, é um diagrama que apresenta a subdivisão dos grupos formados e busca a máxima homogeneidade entre os indivíduos no grupo e máxima heterogeneidade entre os grupos (CRUZ; REGAZZI 1994; MARTEL et al., 2003). Portanto, a análise multivariada permite verificar quais os atributos químicos do solo que expressam maior resposta, eliminando o tempo usado com atributos que expressam baixa resposta (MALUCH-BARRETA et al., 2006).

As técnicas multivariadas permitem avaliar simultaneamente um conjunto de características levando-se em consideração as correlações entre elas. Essas técnicas possibilitam inferências sobre conjuntos de dados no nível de significância conhecido, permitindo assim ampla faixa de compreensão da distribuição geral dos resultados (PAYE et., 2012).

Diante do exposto, o objetivo do presente trabalho foi identificar por meio de análises multivariadas os atributos químicos do solo que melhor explicam a variabilidade da cultura da pupunha em Registro, SP. 


\section{MATERIAL E MÉTODOS}

O experimento foi conduzido na Fazenda de Ensino, Pesquisa e Extensão da Faculdade de Engenharia de Registro, no município de Registro (SP), em Lat. 24\%32'08" e Long. 47 51'55", com uma precipitação média anual é de $1.500 \mathrm{~mm}$, enquanto a sua temperatura média é de $25^{\circ} \mathrm{C}$. O clima é do tipo Af, segundo Köppen-Geiger, caracterizado como tropical sem estação seca.

A caracterização do tipo de solo está definindo como sistema de planícies e terraços fluviais do Ribeira do Iguape, descrito como terreno planos no baixo ribeira e/ou região da depressão tectônica do Ribeira, de sedimentos modernos, em solos aluviais argilosos do tipo Cambissolos Eutróficos em áreas de montante e hidromórficos eutróficos em solos de planície.

O preparo do solo foi realizado por meio de subsolagem em 09 de janeiro de 2012. Em 19 de janeiro de 2012 foi realizado a gradagem aradora. Em 20 de janeiro de 2012 realizou-se a correção do solo com $1,5 \mathrm{tha}^{-1} \mathrm{e}$ em seguida realizada a gradagem niveladora.

A adubação de plantio contou com $150 \mathrm{~g}$ do formulado NPK (4-14-08) e de manutenção foram realizadas de acordo com as análises de solo. Foi aplicado no primeiro ano de plantio a adubação de cobertura com $60 \mathrm{~kg} \mathrm{ha}^{-1} \mathrm{~N} ; 30 \mathrm{~kg} \mathrm{ha}^{-1} \mathrm{P}_{2} \mathrm{O}_{5}$ e $40 \mathrm{~kg} \mathrm{ha}^{-1} \mathrm{~K}_{2} \mathrm{O}+$ $1250 \mathrm{~kg} \mathrm{ha}^{-1}$ de FTE banana (Ca $7 \%$, S 5,7 \%, B $1 \%$, Cu $1 \%$, Mn $1 \%$, Mo 0,1\% e Zn 18 $\%$ ), sendo todas as aplicações ao redor da planta. No segundo ano foi aplicado $100 \mathrm{~kg} \mathrm{ha}^{-1} \mathrm{~N}$, $50 \mathrm{~kg} \mathrm{ha}^{-1} \mathrm{P}_{2} \mathrm{O}_{5}$ e $60 \mathrm{~kg} \mathrm{ha}^{-1} \mathrm{~K}_{2} \mathrm{O}+100 \mathrm{~kg} \mathrm{ha}^{-1}$ de FTE banana e calcário $1000 \mathrm{~kg} \mathrm{ha}^{-1}$. $\mathrm{O}$ plantio foi realizado em 24 de janeiro de 2012, da UNESP Campus Experimental de Registro, SP, e as coletas de planta e solo foram realizadas dia 25 de abril de 2014.

$\mathrm{O}$ espaçamento utilizado para a instalação do experimento foi de 2 x 1 (entrelinhas $\mathrm{x}$ planta). A área contendo 54 pontos foi georreferenciada com o auxílio de um GPS Garmin, de forma a representar toda a área de amostragem de aproximadamente 1 ha, sendo a coleta realizada ao entorno da planta. Para a coleta de atributos da planta utilizou uma régua graduada de $2 \mathrm{~m}$ para determinar a altura. O diâmetro foi coletado a uma altura de $50 \mathrm{~cm}$ do solo com o auxílio de um paquímetro digital. E, para o número de folhas e perfilhos foram feitos a contagem manualmente.

Foram determinados nas amostras de solo a matéria orgânica (MO), $\mathrm{pH}, \mathrm{P}, \mathrm{K}^{+}, \mathrm{Ca}^{2+}$, $\mathrm{Mg}^{2+}, \mathrm{H}+\mathrm{Al}$, soma de bases (SB), capacidade de troca catiônica (CTC), saturação por bases (V\%) e saturação por alumínio (m\%), segundo procedimentos descritos por Raij et al. (1991). As análises químicas foram realizadas no Laboratório de Fertilidade de Solo do Departamento de Fitossanidade, Engenharia Rural e Solos, da Universidade Estadual Paulista (UNESP), Campus de Ilha Solteira.

Foram aplicados três métodos estatísticos multivariados, visando classificar os acessos (cada um dos 54 pontos amostrais) em grupos: análise de agrupamentos hierárquica, análise de agrupamentos não-hierárquica (k-means) e análise de componentes principais (ACP). Todas as análises multivariadas foram realizadas após a padronização das variáveis em que cada uma ficou com média 0 e variância unitária (1).

Primeiramente fez-se a ACP de todas as variáveis de solo e planta estudadas, esses componentes foram construídos pela combinação da correlação entre as variáveis, sendo extraídas em ordem decrescente de importância, em termos de sua contribuição para a variação total dos dados (SILVA et al., 2010), utilizando se o pacote estatístico Statistica.

Estas correlações foram classificadas de acordo com Coelho (2005), onde o critério para classificação dos autovetores (valores que representam o peso de cada caractere, em cada 
componente, e variam de $-1 \mathrm{a}+1)$ foi: valor absoluto $<0,30$, classificado como pouco significativo; 0,30-0,40, considerado mediamente significativo; e $\geq 0,50$, tido como altamente significativo. E então as variáveis que tiveram autovetores com peso $\geq 0,50$ (altamente significativo) foram submetidas a uma nova ACP, para ranquear os componentes com maior carga fatorial (escore).

Posteriormente, os dados foram submetidos ao processamento da análise de agrupamento por método hierárquico adotando a distância euclidiana como medida de semelhança entre as amostras e o método de Ward como método de ligação de grupos. Após adotar uma quantidade de grupos que melhor caracterizou a estrutura de grupos contida nos dados originais foi feita a análise de agrupamento por método não-hierárquico utilizando o algoritmo k-means (HAIR JUNIOR et al., 2005) que permitiu caracterizar o padrão das variáveis por grupo considerado.

\section{RESULTADOS E DISCUSSÃO}

De todos os atributos estudados, sendo eles, número de folhas (NF), diâmetro (Diam), altura (Alt), perfilhos (perf), fósforo (P), matéria orgânica (MO), potencial hidrogeniônico $(\mathrm{pH})$, potássio $(\mathrm{K})$, cálcio $(\mathrm{Ca})$, magnésio $(\mathrm{Mg})$, acidez potencial $(\mathrm{H}+\mathrm{Al})$ e Alumínio $(\mathrm{Al})$, a análise multivariada permitiu que apenas o diâmetro, perfilho, matéria orgânica, potássio, magnésio, alumínio, capacidade de troca catiônica e saturação por alumínio se destacassem como componentes principais.

$\mathrm{Na}$ análise de componentes principais com as variáveis de maiores escores, foram extraídos dois componentes principais que, de forma acumulada, explicam 56,62\% da variabilidade total dos dados, conforme Tabela 1.

Tabela 1. Resumo dos componentes principais (CP1 e CP2) da análise multivariada dos caracteres produtivos da pupunha (Diam e Perf) e de alguns atributos químicos (MO, K, Mg, Al, CTC e m\%) de um Cambissolo eutrófico para a profundidade de $0,00-0,10 \mathrm{~m}$.

\begin{tabular}{ccc}
\hline Componentes Principais & CP1 & CP2 \\
\hline Autovalor & 3,163 & 1,366 \\
VE $(\%)$ & 39,54 & 17,08 \\
\hline Variável & \multicolumn{2}{c}{ Correlação } \\
\hline Diam & 0,119 & $-0,261$ \\
Perf & 0,153 & 0,454 \\
MO & $-0,329$ & $-0,777^{*}$ \\
K & $-0,582^{*}$ & 0,337 \\
Mg & $-0,931^{*}$ & $-0,008$ \\
Al & $0,792^{*}$ & $-0,330$ \\
CTC & $-0,671^{*}$ & $-0,476$ \\
m\% & $0,855^{*}$ & $-0,193$ \\
\hline
\end{tabular}

$\mathrm{VE}=$ Variância explicada. " correlações consideradas na interpretação do componente principal.

O primeiro componente explicou 39,54\% da variabilidade total dos dados da área experimental. Este componente foi constituído pelos atributos potássio, magnésio, alumínio, capacidade de troca catiônica e saturação por alumínio para a profundidade de $0,00-0,10 \mathrm{~m}$, onde o potássio, magnésio e a capacidade de troca catiônica se correlacionaram de maneira negativa, enquanto alumínio e a saturação por alumínio se correlacionaram positivamente. $\mathrm{O}$ segundo componente principal explicou $17,08 \%$ da variabilidade dos dados, sendo constituída apenas pelo atributo matéria orgânica que apresentou correlação negativa. 
A representação gráfica biplot (entre CP1 e CP2) (Figuras 1 e 2) permitiu caracterizar as variáveis que mais discriminaram na formação dos agrupamentos 1 e 2 . Os atributos potássio $(-0,58)$, magnésio $(-0,93)$, capacidade de troca catiônica $(-0,67)$ e matéria orgânica $(-$ $0,33)$ permitiram discriminar o agrupamento 1, localizados à esquerda da CP1 (correlações negativas), enquanto o agrupamento 2 , os atributos responsáveis pela discriminação foram o perfilho $(0,15)$, a saturação por alumínio $(0,85)$, o alumínio $(0,79)$ e o diâmetro do caule da pupunha $(0,12)$. Assim, o agrupamento 1 apresentou os maiores valores de matéria orgânica, potássio, magnésio e capacidade de troca catiônica. Enquanto o agrupamento 2, caracterizouse pelos maiores valores de diâmetro do caule da pupunha, perfilho, alumínio e a saturação por alumínio. A Figura 1 representa a variação espacial no terreno dos dois grupos. Assim, do total de 54 pontos amostrados, 59,26\% representam o agrupamento 1, enquanto, 40,74\% representam o agrupamento 2. Observa-se também que a região sudeste do mapa (Figura 1) encontra-se uma maioria de pontos amostrados representando o agrupamento 2, agrupamento este que obteve o maior diâmetro do caule da pupunha. Enquanto o agrupamento 1, a maioria dos pontos amostrados se encontram ao norte da Figura 1, onde se obteve o menor diâmetro do caule da pupunha.

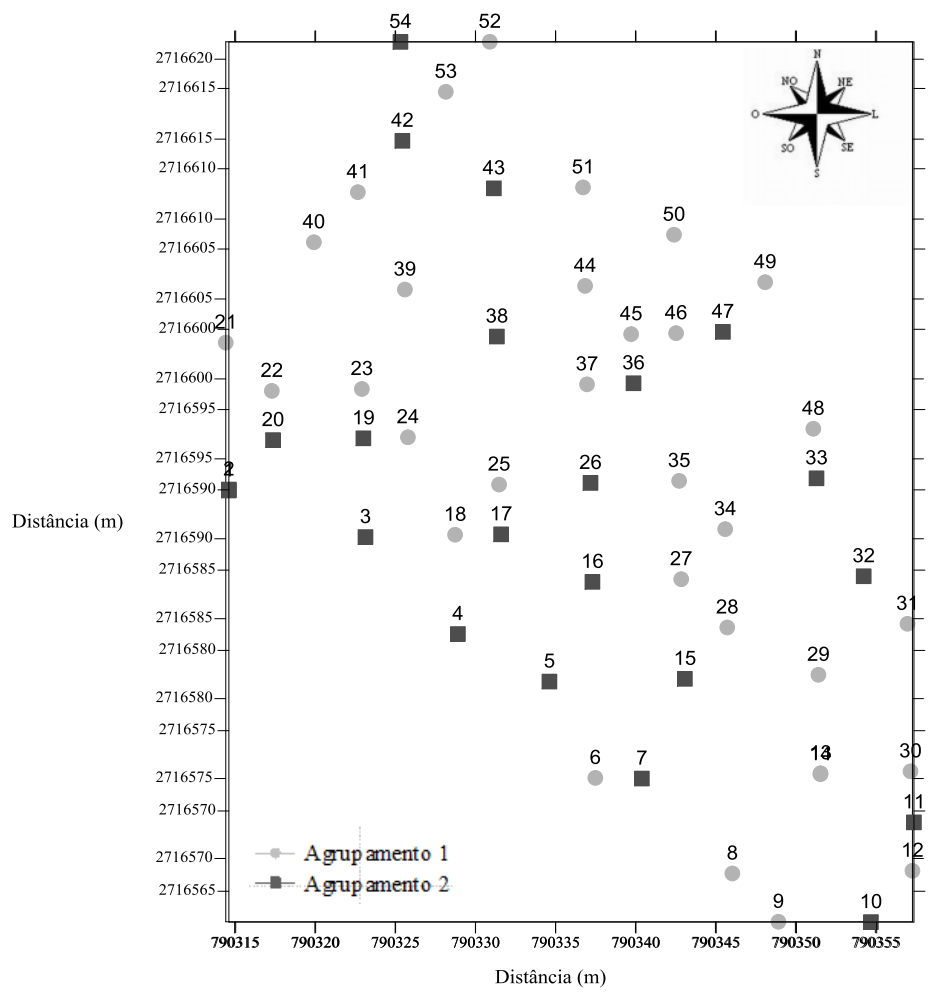

Figura 1. Distribuição espacial dos grupos gerados do diâmetro do caule e Perfilhos da pupunha e de alguns atributos químicos de um Cambissolo eutrófico para a profundidade de $0,00-0,10 \mathrm{~m}$.

O dendrograma (Figura 3) obtido pela análise de agrupamentos hierárquica realizado com os oito componentes principais é apresentado na Figura 3, onde a variação expressiva nos valores de distância euclidiana entre os acessos, para o conjunto de variáveis consideradas, torna possível fazer uma divisão de dois grupos de parcelas homogêneas. Silva et al. (2010) estudando a variabilidade espacial de atributos químicos de um latossolo cultivado com café também encontraram a formação de dois grupos de parcelas homogêneas, quando estudados somente os componentes principais. 


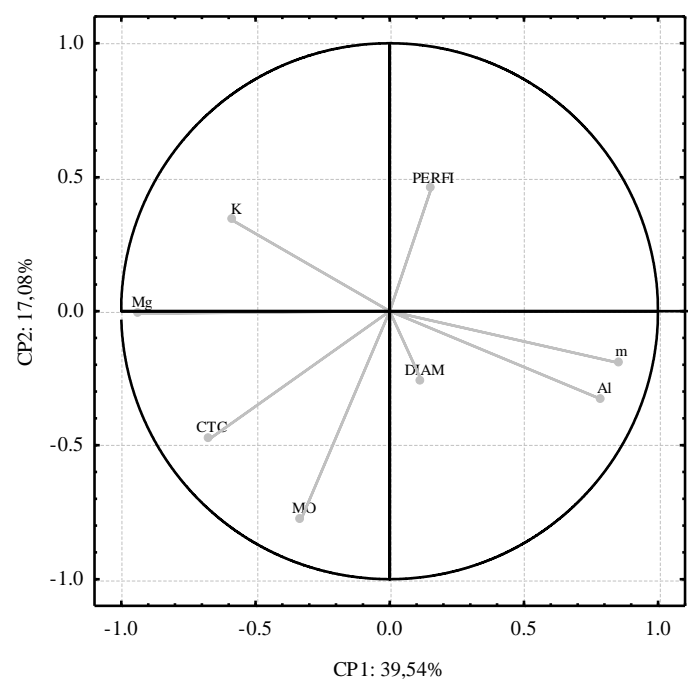

Figura 2. Dispersão (gráfico biplot) dos caracteres dos componentes produtivos da pupunha e de alguns atributos químicos de um Cambissolo eutrófico para a profundidade de $0,00-0,10 \mathrm{~m}$.

A variação encontrada no presente trabalho foi de 18 para 56 e permitiu uma divisão exata dos acessos em dois grupos. Para a confirmação da ordenação obtida na análise de agrupamentos hierárquica, aplicou-se o método de agrupamentos não-hierárquico. Os resultados confirmaram a ordenação e, ainda, conforme análise de variância, todas as variáveis foram importantes para essa ordenação $(p<0,01)$ (Tabela 2).

Tabela 2. Médias dos atributos de planta (Diam e Perf) e solo (MO, K, Mg, Al, CTC e m\%) dos grupos formados pela análise não-hierárquica de agrupamentos k-means.

\begin{tabular}{ccccccccc}
\hline & \multicolumn{7}{c}{ Variáveis } \\
\cline { 2 - 8 } Grupos & $\begin{array}{c}\text { Diam } \\
(\mathrm{cm})\end{array}$ & Perf & $\begin{array}{c}\mathrm{MO} \\
(\mathrm{g} \\
\left.\mathrm{dm}^{-3}\right)\end{array}$ & $\begin{array}{c}\mathrm{K} \\
(\mathrm{mmolc} \\
\left.\mathrm{dm}^{-3}\right)\end{array}$ & $\begin{array}{c}\mathrm{Mg} \\
(\mathrm{mmolc} \\
\left.\mathrm{dm}^{-3}\right)\end{array}$ & $\begin{array}{c}\mathrm{Al} \\
(\mathrm{mmolc} \\
\left.\mathrm{dm}^{-3}\right)\end{array}$ & $\begin{array}{c}\mathrm{CTC} \\
(\mathrm{mmolc} \\
\left.\mathrm{dm}^{-3}\right)\end{array}$ & $\mathrm{m}(\%)$ \\
\hline 1 & 6,1 & 6,8 & 18,2 & 1,5 & 9,5 & 8,3 & 72,6 & 24,7 \\
2 & 6,6 & 6,6 & 19,4 & 2,1 & 25,7 & 1,0 & 85,7 & 1,9 \\
\hline $\begin{array}{c}\text { Média } \\
\text { geral }\end{array}$ & 6,35 & 6,7 & 18,8 & 1,8 & 17,6 & 4,65 & 79,15 & 13,3 \\
\hline $\mathrm{F}$ & 52,49 & 52,97 & $47,28^{*}$ & $47,45^{*}$ & $19,01^{* *}$ & $26,06^{* *}$ & $38,33 * *$ & $22,89 * *$ \\
\hline
\end{tabular}

**: diferença significativa a $0,01 \%$ de probabilidade.

Essa divisão em grupos mostrou um resultado muito importante que foi a ordenação dos acessos segundo o diâmetro do caule da pupunha e o número de perfilhos. Onde, no agrupamento 1 e 2 não houve diferença significativa nos valores de diâmetro do caule da pupunha e o número de perfilhos. Porém para os atributos químicos matéria orgânica, potássio, magnésio e capacidade de troca catiônica obtiveram os maiores valores no agrupamento 2 e os menores valores no agrupamento 1. Já para o alumínio e a saturação por alumínio, foi o inverso, encontrou-se maiores valores no agrupamento 1 e menores valores no agrupamento 2 para a profundidade de 0,00-0,10 m (Figura 4). 


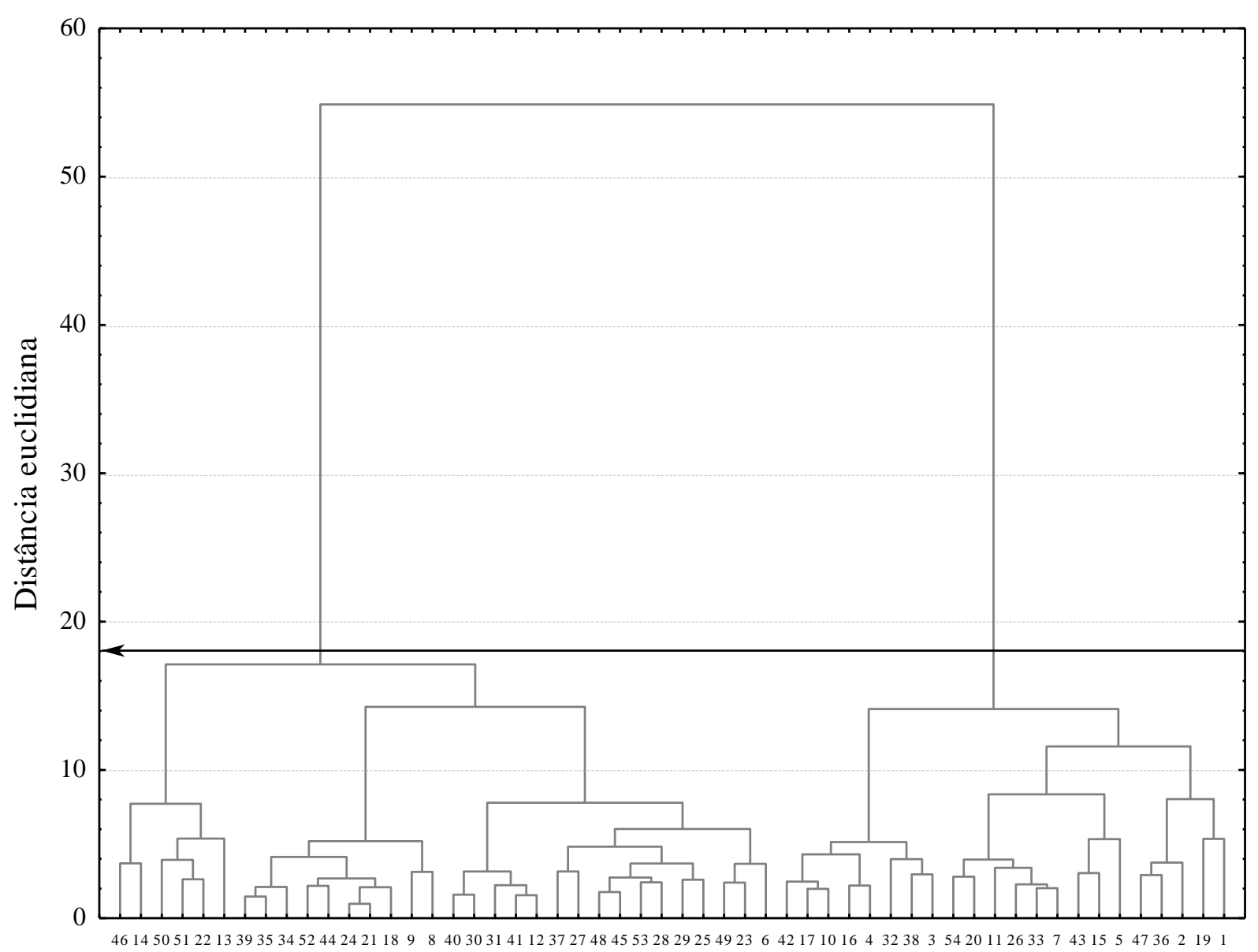

Figura 3. Dendrograma resultante da análise hierárquica de agrupamentos mostrando a formação de grupos segundo o diâmetro do caule e Perfilhos da pupunha, a matéria orgânica, o potássio, o magnésio, o alumínio, a capacidade de troca catiônica e a saturação por alumínio.

Em relação às médias dos atributos químicos (Tabela 2), verificou-se que o solo apresentou nos agrupamentos 1 e 2 valores de teor de matéria orgânica de respectivamente de $18,2 \mathrm{~g} \mathrm{dm}^{-3}$ e $19,4 \mathrm{~g} \mathrm{dm}^{-3}$, valores considerados baixos para solos da região do cerrado (LOPES; COX, 1977). Para o potássio, os valores médios apresentados para cada agrupamento foram obtidos como muito baixa, sendo o ideal acima de 3,0 mmolc $\mathrm{dm}^{-3}$. Já para magnésio os valores obtidos para ambos os agrupamentos foram muitos altos, acima de 4,0 mmolc d $^{-3}$ (RAIJ et al., 1991).

A Figura 4 apresenta as médias padronizadas do diâmetro da pupunha, número de perfilhos e dos atributos químicos do solo para cada grupo, segundo análise de agrupamentos não-hierárquica k-means. Observou-se que, na condição de uma elevada quantidade de matéria orgânica houve-se uma diminuição no teor de potássio. 


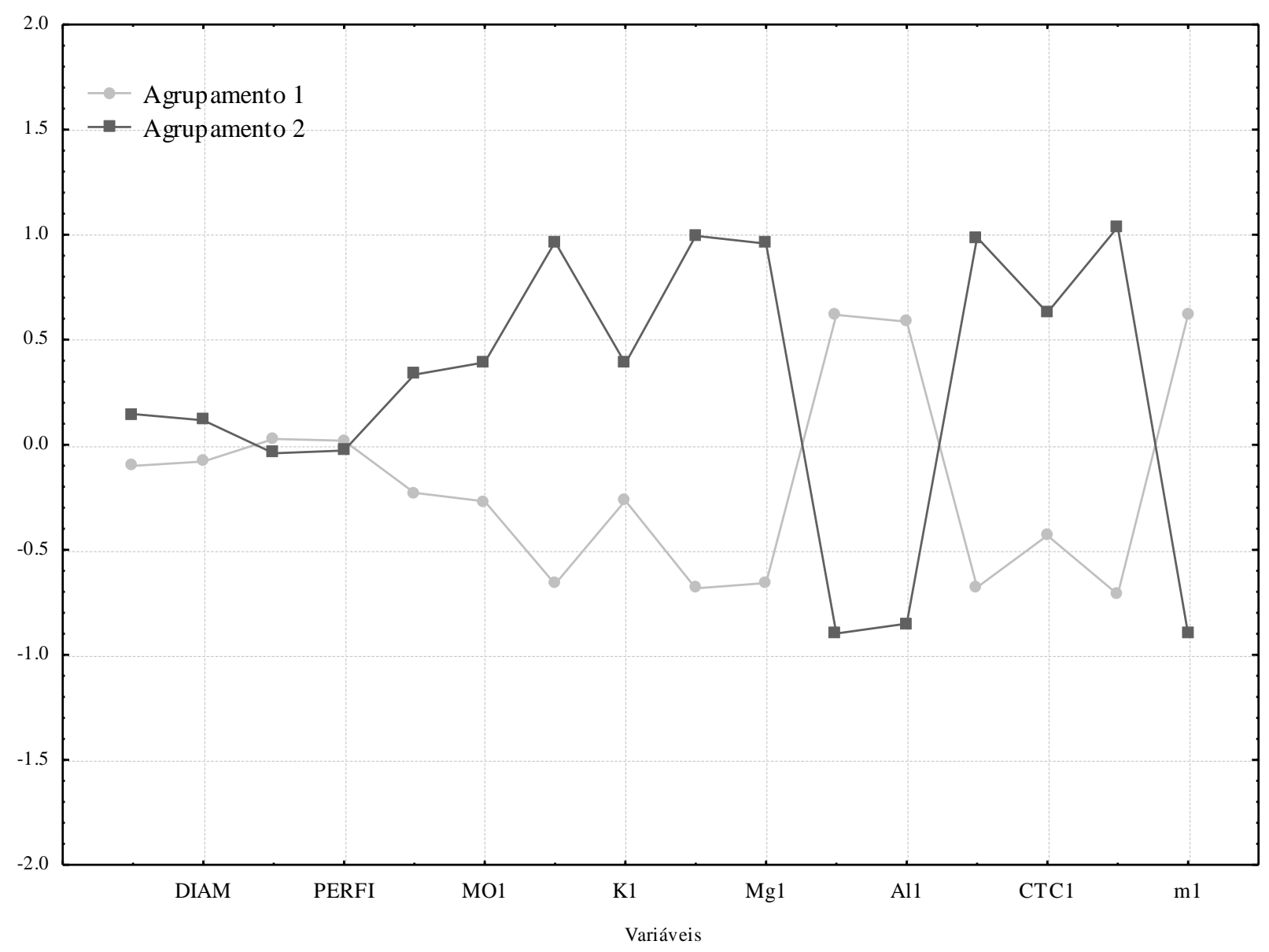

Figura 4. Médias padronizadas do diâmetro do caule e do Perfilhos da pupunha (diam e Perf) e alguns atributos químicos do solo ( $\mathrm{MO}, \mathrm{K}, \mathrm{Mg}, \mathrm{Al}, \mathrm{CTC}$ e m) para cada grupo, segundo análise de agrupamentos não-hierárquica k-means.

\section{CONCLUSÃO}

A análise multivariada permitiu que apenas o diâmetro, perfilho, matéria orgânica, potássio, magnésio, alumínio, capacidade de troca catiônica e saturação por alumínio se destacassem como componentes principais, sendo considerados os mais importantes atributos para o manejo do solo dessa área.

\section{REFERÊNCIAS BIBLIOGRÁFICAS}

BOVI, M. L. A. O agronegócio palmito de pupunha. O Agronômico, Campinas-SP, v. 52, n. 1, p. 10-12, 2000.

COELHO, A. M. Agricultura de precisão: manejo da variabilidade espacial e temporal dos solos e das culturas. In: CURI, N.; MARQUES, J. J. G. S. M.; GUILHERME, L. R. G.; LIMA, J. M.; LOPES, A. S.; VENEGAS, V. H. A. Tópicos em ciência do solo. ViçosaMG: Sociedade Brasileira de Ciência do Solo, 2005. 60 p. V. 1. N. 1.

CORTEZ, J. W.; FERnANDES, A. L. T.; SILVA, R. P.; CARVAlHO FILHO, A.; FURLANI, C. E. A. Métodos multivariados na avaliação dos atributos físicos do solo na cafeicultura irrigada. Engenharia na agricultura, Viçosa-MG, v. 19, n. 2, p. 132-140, 2011. 
CRUZ, C. D.; REGAZZI, A. J. Modelos biométricos aplicados ao melhoramento genético. Viçosa-MG: Universidade Federal de Viçosa, 1994. 390 p. V. 2. N. 2.

FRAZÃO, L. A.; PÍCCOLO, M. C.; FEIG, B. J.; CERRI, C. C.; CERRI, C. E. P. Propriedades químicas de um Neossolo Quartzarênico sob diferentes sistemas de manejo no Cerrado mato-grossense. Pesquisa Agropecuária Brasileira, Brasília-DF, v. 43, n. 5, p. 641648, 2008.

HAIR JUNIOR, J. F.; ANDERSON R. E.; TATHAM, R. L.; BLACK, W. C. Análise multivariada de dados. 5. ed. Porto Alegre-RS: Bookman, 2005. 597 p.

IBGE - INSTITUTO BRASILEIRO DE GEOGRAFIA E ESTATÍSTICA. Censo agropecuário - Resultados preliminares, Rio de Janeiro. 2009.

LOPES, A. S.; COX, F. R. A survey of the fertility status of surface soils under cerrado vegetation in Brazil. Soil Science Society of America Journal, Madison-USA, v. 41, n. 1, p.742-747, 1977.

LOURENTE, E R. P.; LOURENTE, E. R. P.; MERCANTE, F. M.; ALOVISI, A. M. T.; GOMES, C. F.; GASPARINI, A. S.; NUNES, C. M. Atributos microbiológicos, químicos e físicos de solo sob diferentes sistemas de manejo e condições de cerrado. Pesquisa Agropecuária Tropical, Goiânia-GO, v. 41, n. 1, p. 20-28, 2011.

MALUCHE-BARETTA, C. R. D.; AMARANTE, C. V. T; KLAUBERG FILHO, O. Análise multivariada de atributos do solo em sistemas convencional e orgânico de produção de maçãs. Pesquisa Agropecuária Brasileira, Brasília-DF, v. 41, n. 10, p. 1531-1539, 2006.

MARTEL, J. H. I.; FERRAUDO, A. S.; MORÔ, J. R.; PERECIN, D. Estatística multivariada na discriminação de raças amazônicas de pupunheiras (Bactris gasipaes Kunth) em Manaus (Brasil). Revista Brasileira de Fruticultura, Jaboticabal-SP, v. 25, n. 1, p. 115-118, 2003.

PAYE, H. S.; MEllo, J. W. V.; MELO, S. B. Métodos de Análise Multivariada no Estabelecimento de Valores de Referência de Qualidade para Elementos-Traço em Solos. Revista Brasileira de Ciência do Solo, Viçosa-MG, v. 36, n. 3, p. 1031-1041, 2012.

RAIJ, E. V.; ANDRADE, J. C.; CANTARELLA, H.; QUAGGIO, J. A. Análise química para avaliação da fertilidade de solos tropicais. Campinas-SP: Instituto Agronômico de Campinas, Campinas-SP, v. 1, n. 1, p. 28-42, 1991.

SILVA, A. S.; LIMA, J. S. S.; XAVIER, A. C.; TEIXEIRA, M. M. Variabilidade espacial de atributos químicos de um Latossolo Vermelho-amarelo húmico cultivado com café. Revista Brasileira de Ciência do Solo, Viçosa-MG, v. 34, n. 1, p. 15-22, 2010.

VALENTIN, J. L. Ecologia numérica: uma introdução à análise multivariada de dados ecológicos. Rio de Janeiro-RJ: Interciência, 2000. 117 p. V. 1. N. 1. 